\title{
ON LOCALIZING GLOBAL PARETO SOLUTIONS IN A GIVEN CONVEX SET
}

\begin{abstract}
Sufficient conditions are given for the global Pareto solution of the multicriterial optimization problem to be in a given convex subset of the domain. In the case of maximizing real valued-functions, the conditions are sufficient and necessary without any convexity type assumptions imposed on the function. In the case of linearly scalarized vector-valued functions the conditions are sufficient and necessary provided that both the function is concave and the scalarization is increasing with respect to the cone generating the preference relation.
\end{abstract}

1. Introduction. The aim of this paper is to investigate a sufficient and necessary condition for a vector-valued function $g$ to attain its weak Pareto maximum in a given convex subset $V$ of a vector space $X$. Seemingly, the condition is somehow similar to that of Pshenichny necessary and sufficient conditions for a continuous and concave real function to attain its maximum at a given point $x \in X$ in terms of the subgradient and of the cone of feasible directions. Pshenichnyı's result was extended by Swartz (see [9]) to the case of Pareto maxima for vector-valued functions.

Comparing with the Pshenichny $\breve{1}$ and Swartz results, we do not assume that $g$ is continuous. Moreover, we give a sufficient and necessary condition for the weak Pareto maximum of $g$ to be attained on a given set $V \subseteq X$ not necessarily at a given point of $X$. Even when $V$ consists of one point only, our condition differs from those of Pshenichny $\breve{1}$ and Swartz. This can be easily seen in the case of real-valued functions where our condition is sufficient and necessary without imposing any convexity type conditions on $g$, contrary to the result of Pshenichny $\breve{1}$, which does not hold without such assumptions.

1991 Mathematics Subject Classification: 49K27, 49K30, 49J52, 49M49.

Key words and phrases: Pareto optimal solutions, feasible directions, dual cones, sufficient and necessary conditions for optimality. 
We first fix the notation. Let $X$ and $Z$ be real, locally convex topological vector spaces, and let $K$ be a closed convex cone in $Z$. Let $K^{0}$ denote the interior of $K$. We assume throughout the paper that $K^{0} \neq \emptyset$. Let $\Omega$ be a subset of $X$, and $f: \Omega \rightarrow \mathbb{R}$ be a given function.

Fix a nonempty convex set $V \subset X$ and a point $x \notin V$. Let $\partial f(V, x) \subset X^{\prime}$ (the dual space to $X$ ) be the set of all $x^{\prime} \in X^{\prime}$ such that

$$
\exists v \in V, t \in(0,1]: \quad f\left(x_{t}\right)-f(x) \geq\left\langle x^{\prime}, x_{t}-x\right\rangle,
$$

where $x_{t}=t v+(1-t) x$, i.e. $x_{t}-x=t(v-x)$. Clearly, $\partial f(V, x) \supseteq \partial f(W, x)$ for any $V \supseteq W$ and $x \in X$.

Recall that the subdifferential $\partial f(x)$ of $f$ at $x$ is defined as the set of all $x^{\prime} \in X^{\prime}$ satisfying

$$
f(y)-f(x) \geq\left\langle x^{\prime}, y-x\right\rangle \quad \text { for all } y \in \Omega .
$$

Observe that $\partial f(V, x) \supseteq \partial f(x)$ for any $\emptyset \neq V \subseteq \Omega, x \in \Omega \backslash V$ and $f: \Omega \rightarrow \mathbb{R}$.

For $x \notin V$, denote by $G(V, x)$ the set of all directions leading to $V$ from $x$, i.e.

$$
G(V, x)=\{u \in X: x+t u \in V \text { for some } t>0\} .
$$

Clearly, $G(V, x)=\{t(v-x): v \in V$ and $t>0\}$ and because $V$ is convex, $G(V, x)$ is a convex cone as well.

Let $G(V, x)^{*}$ denote the dual cone of $G(V, x)$, i.e.

$$
G(V, x)^{*}=\left\{x^{\prime} \in X^{\prime}:\left\langle x^{\prime}, u\right\rangle \geq 0 \text { for all } u \in G(V, x)\right\} .
$$

A function $g: \Omega \rightarrow Z$ has a weak Pareto maximum (weak $P$-maximum) at $x \in \Omega$ if there exists no $y \in \Omega$ such that $g(y)-g(x) \in K^{0}$.

A function $\varphi: Z \rightarrow \mathbb{R}$ is strictly monotone with respect to the cone $K^{0}$ if for every $x, y \in X$ such that $y \in x+K^{0}$ we have $\varphi(y)>\varphi(x)$.

Let us recall Pshenichnyı's condition. It states that a continuous concave function $f$ attains its maximum over a convex set $\Omega$ at $x \in \Omega$ iff

$$
\partial[-f](x) \cap F(\Omega, x)^{*} \neq \emptyset,
$$

where $F(\Omega, x)^{*}$ is the dual cone of $F(\Omega, x)=\{u \in X: \exists \tau>0$ such that $x+t u \in \Omega$ for all $0 \leq t \leq \tau\}$, the cone of feasible directions to $\Omega$ at $x \in \Omega$.

Let $V$ be a convex sequentially compact subset of $\Omega$ such that $\overline{\Omega \backslash V} \subseteq$ $\Omega$. The main result of the paper is Theorem 3.1, which says that if there is a function $\varphi: Z \rightarrow \mathbb{R}$ which is strictly monotone with respect to $K^{0}$ and such that $\varphi \circ g$ is upper semicontinuous on $\Omega$ and for every $x \in \Omega \backslash V$,

$$
\partial(\varphi \circ g)(V, x) \cap G(V, x)^{*} \neq \emptyset,
$$

then $g$ attains its weak $P$-maximum over $\Omega$ in $V$. The result need not hold when: 1) $\varphi \circ g$ is not upper semicontinuous, or 2) $\overline{\Omega \backslash V} \nsubseteq \Omega$, or 3) $V$ is not a convex set, or 4) $V$ is not sequentially compact, or 5) $\emptyset=$ $\partial(\varphi \circ g)(V, x) \cap G(V, x)^{*}$ (see Examples 3.8-3.10 in Section 3). 
The sufficient condition turns out to be necessary provided $g$ is a realvalued function, no matter if $g$ is concave or not (Proposition 3.6). The condition is no longer necessary if $g$ is vector-valued and $\varphi$ is linear (Example 3.4). When $g$ is $K$-concave, i.e.

$-g(t x+(1-t) y)+\operatorname{tg}(x)+(1-t) g(y) \in-K \quad$ for $x, y \in \Omega$ and $t \in[0,1]$, and $\varphi$ is linear the condition becomes again necessary (Proposition 3.7).

An open question is what other kind of correspondence between $g$ and $\varphi$ makes Theorem 3.1 a sufficient and necessary condition for a weak $P$ maximum of $g$ to be in $V$.

2. Preliminary notions and results. To prove the main result we need some results on sequentially compact sets and countably orderable sets. Therefore we first recall some notions and results from [2], [4] and [8].

Let $X$ be a nonempty set and $s \subset X^{2}$ be an arbitrary relation in $X$. We write $x s y$ instead of $(x, y) \in s$; by $s^{*}$ we mean the transitive closure of $s$, i.e. $x s^{*} y$ iff $x=x_{1} s \ldots s x_{n}=y$ for some finite sequence $x_{1}, \ldots, x_{n} \in X$; by $\sim(x s y)$ we mean $(x, y) \notin s$. For every $x \in X$ and $U \subseteq X$ we denote by $U_{s}(x)$ the set $\{z \in U: x s z\}$. If $X$ is a linear space, for every $x, y \in X,[x, y]$ means the set $\{z \in X: \exists t \in[0,1]$ such that $z=t x+(1-t) y\}$.

Definition 2.1 (see [4, p. 288]). $X$ is called countably orderable with respect to the relation $s$ if for every nonempty subset $W \subseteq X$ the existence of a relation $\eta$ well ordering $W$ and such that $\eta \subseteq s^{*} \cup$ id implies that $W$ is at most countable.

A number of examples of countably orderable sets together with applications to optimization are given in [4].

The following result has been proven in [4].

TheOREM 2.2 (see [4, Theorem 4.1, p. 297]). Let $X$ be a nonempty set with relations s, $r \subseteq X \times X$. Let $U, V \subseteq X$ be nonempty sets such that $U \backslash V \neq \emptyset, U \backslash V$ is countably orderable with respect to $r$ and $r$ is transitive on $U \backslash V$. Assume that for every $u \in U \backslash V$ the set $U_{s}(u)$ is nonempty and the following conditions hold:

(i) for every sequence $\left(x_{i}\right) \subseteq U_{s}(u) \backslash V$ such that $x_{i} r x_{i+1}$ for every $i \in \mathbb{N}$ there is $x \in U_{s}(u)$ with $x_{i} r x$ for every $i \in \mathbb{N}$;

(ii) for every $x \in U_{s}(u) \backslash V$ there is $y \in U_{s}(u)$ for which $x r y, x \neq y$ and $\sim(y r x)$.

Then $U \cap V \neq \emptyset$ and for every $u \in U \backslash V$ there exists $v \in U \cap V$ such that usv.

Now we recall the definition of a sequentially compact set. 
Definition 2.3 (see [2, p. 261]). A nonempty subset $A$ of a topological Hausdorff space $X$ is called sequentially compact if for every sequence $\left\{x_{i}\right\}$ in $A$ there is a subsequence $\left\{x_{i_{k}}\right\} \subseteq\left\{x_{i}\right\}$ converging to some $x \in A$.

The following results have been given in [2].

TheOREM 2.4 (see [2, Theorem 24, p. 262]). The Cartesian product of a countable number of sequentially compact spaces is sequentially compact with Tikhonov's topology.

TheOrem 2.5 (see [2, Theorem 21, p. 262]). Let $X$ be a sequentially compact space. If there is a continuous function $f$ from $X$ onto $Y$ and $Y$ is a Hausdorff space, then $Y$ is sequentially compact.

Let $X$ be a vector space and $A$ be a subset of $X$.

DEFinition 2.6 (see [8, p. 47]). The intersection of all convex sets containing $A$ is called the convex hull $(\operatorname{conv}(A))$ of $A$.

It is easy to check that

$$
\begin{aligned}
\operatorname{conv}(A)= & \{y \in X: \\
& \left.y=\sum_{i=1}^{n} \alpha_{i} a_{i}, a_{i} \in A, \alpha_{i} \geq 0, \sum_{i=1}^{n} \alpha_{i}=1, n \text { arbitrary }\right\} .
\end{aligned}
$$

Lemma 2.7. Let $A$ be a sequentially compact and convex subset of a real locally convex topological space $X$ and $x_{0} \in X$. Then $\operatorname{conv}\left(A \cup\left\{x_{0}\right\}\right)$ is sequentially compact.

Pr o of. Since $A$ is convex, it follows from (2) that

$$
\begin{aligned}
\operatorname{conv}\left(A \cup\left\{x_{0}\right\}\right)= & \{y \in X: \\
& \left.y=\sum_{i=1}^{2} \alpha_{i} a_{i}, a_{1} \in A, a_{2}=x_{0}, \alpha_{1}+\alpha_{2}=1, \alpha_{1}, \alpha_{2} \geq 0\right\} .
\end{aligned}
$$

Define

$$
L:=\left\{\left(\alpha_{1}, \alpha_{2}\right): \alpha_{1}, \alpha_{2} \geq 0 \text { and } \alpha_{1}+\alpha_{2}=1\right\} \subset \mathbb{R}^{2} .
$$

It is easy to show that $L$ is a sequentially compact subset of $\mathbb{R}^{2}$. Observe that $\operatorname{conv}\left(A \cup\left\{x_{0}\right\}\right)$ is a continuous image of the sequentially compact set $L \times A \times\left\{x_{0}\right\} \subset \mathbb{R}^{2} \times X \times X$ (see Theorem 2.4), so by Theorem 2.5, it is sequentially compact.

3. The main results. We start this section with presenting a general sufficient condition for a global Pareto maximum of a vector-valued function to be in a given convex subset of the domain. 
TheOREM 3.1. Let $X$ and $Z$ be locally convex topological vector spaces, $\Omega$ be a nonempty subset of $X$ and $g: \Omega \rightarrow Z$. Let $V \neq \emptyset$ be a given sequentially compact, convex subset of $\Omega$ such that $\overline{\Omega \backslash V} \subseteq \Omega$. If a function $\varphi: g(\Omega) \rightarrow \mathbb{R}$ is such that $\varphi \circ g$ is upper semicontinuous on $\overline{\Omega \backslash V}$ and

$$
\emptyset \neq \partial(\varphi \circ g)(V, y) \cap G(V, y)^{*}
$$

for every $y \in \Omega \backslash V$, then

$$
\sup _{x \in \Omega} \varphi \circ g(x)=\sup _{v \in V} \varphi \circ g(v) .
$$

If , additionally, $\varphi$ is strictly monotone with respect to $K^{0}$ and $\varphi \circ g$ attains its maximum over $V$ at $v_{0} \in V$, then $v_{0}$ is a global weak $P$-maximum of $g$ over $\Omega$.

Proof. Fix $\varepsilon>0$ and a convex absorbing neighbourhood $U$ of zero. Let $\mu_{U}$ denote the Minkowski functional relative to $U$. Define a relation $s \subset \Omega \times \Omega$ by

$$
\forall x, y \in \Omega: \quad x s y \Leftrightarrow \varphi \circ g(x)-\varepsilon \mu_{U}(x, V) \leq \varphi \circ g(y)-\varepsilon \mu_{U}(y, V),
$$

where $\mu_{U}(x, V)=\inf _{v \in V} \mu_{U}(v-x)$. Define a relation $r \subset \Omega \times \Omega$ by

$$
\forall x, y \in \Omega: \quad x r y \Leftrightarrow \exists v \in V: y \in[v, x] .
$$

Since $V$ is convex, $r$ is transitive.

When $\Omega \backslash V=\emptyset$ the assertion trivially holds, so assume that $\Omega \backslash V \neq \emptyset$. First we show that $\Omega \backslash V$ is countably orderable with respect to $r$. Let $W \subseteq \Omega \backslash V$ be well ordered with respect to some relation $\eta \subseteq r \cup$ id. Let $w_{0}$ be the $\eta$-first element of $W$ and denote $V-\operatorname{conv}\left(V \cup\left\{w_{0}\right\}\right)$ by $A$. Clearly, $t A \subseteq A$ for every $t \in[0,1]$. Since $A$ is convex and absorbing (for $V-W$ ), it is easy to prove that the Minkowski functional of $A$ has the following properties (cf. [8]):

$1^{\circ} \mu_{A}(x)<\infty$ for all $x \in V-W$,

$2^{\circ} \mu_{A}(x+y) \leq \mu_{A}(x)+\mu_{A}(y)$ for all $x, y \in V-W$,

$3^{\circ} \mu_{A}(\lambda x)=\lambda \mu_{A}(x)$ for all $x \in V-W$ and $\lambda \in \mathbb{R}_{+}$.

From Lemma 2.7 it also follows that $A$ is sequentially compact.

Now we show, using the above properties of $A$ and $\mu_{A}$, that the conditions $x, y \in W, x \neq y$ and $x \eta y$ together force

$$
\mu_{A}(x, V)>\mu_{A}(y, V),
$$

where $\mu_{A}(x, V)=\inf _{v \in V} \mu_{A}(v-x)$. First we show that $\mu_{A}(x, V)>0$ for all $x \in W$. Suppose that $\mu_{A}(x, V)=0$ for some $x \in W$. Then for every $n>0$ there is $v_{n} \in V$ such that $\mu_{A}\left(v_{n}-x\right)<2^{-n}$, hence $v_{n}-x \in 2^{-n} A$. Now suppose that $x$ is not the limit of $\left\{v_{n}\right\}$. Then there is a convex symmetric neighbourhood $U_{0}$ of zero and a subsequence $\left\{v_{n_{k}}\right\} \subseteq\left\{v_{n}\right\}$ such that $v_{n_{k}}-$ $x \notin U_{0}$ for all $n_{k}$. The way the sequence $\left\{v_{n}\right\}$ was constructed implies that 
we can choose points $a_{n_{k}} \in A$ for which $v_{n_{k}}-x=2^{-n_{k}} a_{n_{k}} \notin U_{0}$ for every $k \in \mathbb{N}$. On the other hand, $A$ is sequentially compact, so $a_{n_{k_{j}}} \rightarrow a \in A$ for some subsequence $\left\{a_{n_{k_{j}}}\right\}$, and hence $\mu_{U_{0}}\left(a_{n_{k_{j}}}-a\right) \leq 1$ for every $j$ large enough. Thus for $j \rightarrow \infty$,

$$
\infty \leftarrow 2^{n_{k_{j}}} \leq \mu_{U_{0}}\left(a_{n_{k_{j}}}\right) \leq \mu_{U_{0}}(a)+\mu_{U_{0}}\left(a_{n_{k_{j}}}-a\right) \leq \mu_{U_{0}}(a)+1<\infty,
$$

which is a contradiction. Therefore the sequence $\left\{v_{n}\right\}$ converges to $x$. Since $V$ is sequentially compact, we have $x \in V$, which is a contradiction because $x \in W \subseteq \Omega \backslash V$. So $\mu_{A}(x, V)>0$ for all $x \in W$.

Now let $x, y \in W, x \neq y$ and $x \eta y$. Then there are $t \in(0,1)$ and $u \in V$ such that $y=(1-t) x+t u$. We can take $v \in V$ such that

$$
\mu_{A}(v-x)<(1+t) \mu_{A}(x, V) .
$$

Observe that since $V$ is convex, we have $(1-t) v+t u \in V$ and by $3^{\circ}$,

$$
\begin{aligned}
\mu_{A}(y, V) & \leq \mu_{A}((1-t) v+t u-y) \\
& =\mu_{A}((1-t) v+t u-(1-t) x-t u) \\
& =(1-t) \mu_{A}(v-x)<(1-t)(1+t) \mu_{A}(x, V)<\mu_{A}(x, V) .
\end{aligned}
$$

Thus the set $\mu_{A}(W, V) \subseteq \mathbb{R}$ is well ordered by $\leq$ and therefore it is at most countable. But the same concerns $W$, since $\mu_{A}(\cdot, V)$ is a one-to-one mapping on $W$. So $\Omega \backslash V$ is countably orderable with respect to $r$.

Now, fix $x_{0} \in \Omega \backslash V$ and let $\Omega_{s}\left(x_{0}\right)=\left\{x \in \Omega: x_{0} s x\right\}$. Consider a sequence $\left\{x_{i}\right\} \subset \Omega_{s}\left(x_{0}\right) \backslash V$ such that $x_{i} r x_{i+1}$ for all $i=1,2, \ldots$ Since $r$ is transitive, $x_{i} \in \operatorname{conv}\left(V \cup\left\{x_{1}\right\}\right)$ for all $i=2,3, \ldots$, and from Lemma 2.7, $\operatorname{conv}\left(V \cup\left\{x_{1}\right\}\right)$ is sequentially compact. Choose a subsequence $\left\{x_{i_{k}}\right\} \subseteq\left\{x_{i}\right\}$ converging to some $x \in \operatorname{conv}\left(V \cup\left\{x_{1}\right\}\right)$. Then $x_{i_{k+j}} \in \operatorname{conv}\left(V \cup\left\{x_{i_{k}}\right\}\right)$ for every $j=1,2, \ldots$ Therefore $x \in \operatorname{conv}\left(V \cup\left\{x_{i_{k}}\right\}\right)$ for all $k=1,2, \ldots$ and $x_{i_{k}} r x$ for all $k=1,2, \ldots$

Now, we show that $\mu_{U}(x, V) \geq \mu_{U}(y, V)$ for all $x, y \in \Omega \backslash V$ with $x \neq y$ and $x r y$.

$1^{\circ}$ If $\mu_{U}(x, V)=0$ and $x \in \Omega \backslash V$, then for every $n \in \mathbb{N}$ there exists $v_{n} \in V$ such that $\mu_{U}\left(v_{n}-x\right)<2^{-n}$. Since $V$ is sequentially compact there is a subsequence $\left\{v_{n_{k}}\right\} \subseteq\left\{v_{n}\right\}$ converging to a point $v_{0} \in V$. Consequently, for every $n_{0} \in \mathbb{N}$, there is $k \geq n_{0}$ such that for $n_{j}>k$ we have $v_{n_{j}} \in 2^{-n_{0}} U+v_{0}$. Hence for $n_{0} \rightarrow \infty$,

$$
0 \leq \mu_{U}\left(v_{0}-x\right) \leq \mu_{U}\left(v_{0}-v_{n_{j}}\right)+\mu_{U}\left(v_{n_{j}}-x\right) \leq \frac{1}{2^{n_{0}}}+\frac{1}{2^{n_{j}}} \rightarrow 0 .
$$

So there is $v_{0} \in V$ such that $\mu_{U}\left(v_{0}-x\right)=0$, and finally,

$$
\mu_{U}\left(v_{0}-x\right)=\mu_{U}(x, V)=0 .
$$


$2^{\circ}$ If $\mu_{U}(x, V)>0$, then

$$
\forall t \in(0,1) \exists v_{0} \in V: \quad \mu_{U}\left(v_{0}-x\right)<(1+t) \mu_{U}(x, V) .
$$

From (4) and (5), we get in both cases $1^{\circ}$ and $2^{\circ}$,

(6) $\forall x \in \Omega \backslash V, t \in(0,1) \exists v_{0} \in V: \quad \mu_{U}\left(v_{0}-x\right) \leq(1+t) \mu_{U}(x, V)$.

Let $x, y \in \Omega \backslash V, x \neq y$ and $x r y$. By the definition of $r$, there are $t \in(0,1)$ and $u \in V$ such that $y=t u+(1-t) x$. From (6) and the fact that $V$ is convex, it follows that $t u+(1-t) v_{0} \in V$ and

(7) $\mu_{U}(y, V) \leq \mu_{U}\left(\left(t u+(1-t) v_{0}-t u-(1-t) x\right)\right.$

$$
=(1-t) \mu_{U}\left(v_{0}-x\right) \leq(1-t)(1+t) \mu_{U}(x, V) \leq \mu_{U}(x, V) .
$$

Since $x_{0} s x_{i_{k}}$ for all $k=1,2, \ldots$ and $\varphi \circ g$ is upper semicontinuous on $\overline{\Omega \backslash V}$, by (7) we get

$$
\begin{aligned}
\varphi \circ g\left(x_{0}\right)-\varepsilon \mu_{U}\left(x_{0}, V\right) & \leq \varphi \circ g\left(x_{i_{k}}\right)-\varepsilon \mu_{U}\left(x_{i_{k}}, V\right) \\
& \leq \varphi \circ g(x)-\varepsilon \mu_{U}(x, V)
\end{aligned}
$$

whenever $x \notin V$. Otherwise $0=\mu_{U}(x, V) \leq \mu_{U}\left(x_{i_{k}}, V\right)$, and the above inequality holds as well. This shows that $x \in \Omega_{s}\left(x_{0}\right)$ and the condition (i) of Theorem 2.2 is satisfied.

Now we examine the condition (ii). Consider any $x \in \Omega_{s}\left(x_{0}\right) \backslash V$ and observe that there are, by $(3)$, an $x^{\prime} \in \partial(\varphi \circ g)(V, x)$ and an $v \in V$ such that

$$
\varphi \circ g\left(x_{t}\right)-\varphi \circ g(x) \geq\left\langle x^{\prime}, x_{t}-x\right\rangle \quad \text { for some } t \in(0,1]
$$

where $x_{t}=t v+(1-t) x$. Simultaneously, $x^{\prime} \in G(V, x)^{*}$ and since $x_{t}-x=$ $t(v-x) \in G(V, x)$ we get

$$
\left\langle x^{\prime}, x_{t}-x\right\rangle \geq 0
$$

From (8) and (9), it follows that

$$
\varphi \circ g\left(x_{t}\right)-\varphi \circ g(x) \geq 0 \geq-t^{2} \varepsilon \mu_{U}(x, V) .
$$

It is easy to check (cf. (7)) that

$$
\mu_{U}\left(x_{t}, V\right) \leq\left(1-t^{2}\right) \mu_{U}(x, V) .
$$

From (10) and (11), we get

$$
\varphi \circ g\left(x_{t}\right)-\varphi \circ g(x) \geq-t^{2} \varepsilon \mu_{U}(x, V) \geq \varepsilon \mu_{U}\left(x_{t}, V\right)-\varepsilon \mu_{U}(x, V) .
$$

Consequently,

$$
\varphi \circ g\left(x_{t}\right)-\varepsilon \mu_{U}\left(x_{t}, V\right) \geq \varphi \circ g(x)-\varepsilon \mu_{U}(x, V) .
$$

Thus $x r x_{t}, x \neq x_{t}$ and $x_{t} \in \Omega_{s}\left(x_{0}\right)$ (recall that $s$ is transitive), which shows that (ii) holds. Therefore Theorem 2.2 implies that

$$
\forall x \in \Omega \backslash V \exists v \in \Omega \cap V: \quad x s v .
$$


So we have

$\forall x \in \Omega \backslash V \exists v \in V:$

$$
\varphi \circ g(x)-\varepsilon \mu_{U}(x, V) \leq \varphi \circ g(v)-\varepsilon \mu_{U}(v, V)=\varphi \circ g(v) \leq \sup _{v \in V} \varphi \circ g(v) .
$$

Since $\varepsilon>0$ is arbitrary, the first assertion of the theorem holds.

Assume now that $\varphi \circ g$ attains its maximum over $V$ at $v_{0} \in V$. Then it follows from the first part of Theorem 3.1 that

$$
\forall x \in \Omega: \quad \varphi \circ g(x)-\varphi \circ g\left(v_{0}\right) \leq 0 .
$$

If $v_{0}$ were not a weak $P$-maximum of $g$ over $\Omega$, there would exist $y \in \Omega$ such that

$$
g(y)-g\left(v_{0}\right) \in K^{0} .
$$

Since $\varphi$ is strictly monotone with respect to $K^{0}$, this and (12) would imply

$$
0<\varphi \circ g(y)-\varphi \circ g\left(v_{0}\right) \leq 0,
$$

which is a contradiction. Therefore $g$ has a weak $P$-maximum over $\Omega$ at $v_{0} \in V$.

REMARK 3.2. If we assume that $\varphi \circ g$ is upper semicontinuous on $V$, then $\varphi \circ g$ attains its maximum over $V$ at some $v_{0} \in V$. Moreover, one can only assume increasing semicontinuity of $\varphi \circ g$ (instead of upper semicontinuity) (see [5]) and the same assertion holds. Indeed, this is a consequence of the following lemma.

Lemma 3.3 [4]. Let $X$ be an abstract set and $f: X \rightarrow \mathbb{R}$. The function $f$ attains its supremum at some point of $X$ if and only if for every sequence $\left\{x_{i}\right\} \subseteq X$ such that for every $i=1,2, \ldots$,

$$
f\left(x_{i}\right) \leq f\left(x_{i+1}\right)
$$

there is an $x \in X$ such that $f\left(x_{i}\right) \leq f(x)$ for all $i=1,2, \ldots$

The following example shows that Theorem 3.1 does not give necessary conditions for a weak $P$-maximum of $g$ to be in $V$ when $Z$ is more than one-dimensional and $\varphi$ is linear and strictly monotone with respect to $K^{0}$.

EXAMPLE 3.4. Let $K^{0}$ be the cone of vectors in $\mathbb{R}^{2}$ with positive coordinates. Define $g: \Omega \equiv[1,2] \rightarrow \mathbb{R}^{2}$ by $g(x)=(x, 1 / x)$ and $\varphi_{\varepsilon}: \mathbb{R}^{2} \rightarrow \mathbb{R}$ by $\varphi_{\varepsilon}(x, y)=\varepsilon x+(1-\varepsilon) y$ with $\varepsilon \in[0,1]$. Set $V=\{3 / 2\}$. It is easy to see that $x_{0}=3 / 2$ is a weak $P$-maximum. Now we show that for every $\varphi_{\varepsilon}, \varepsilon \in[0,1]$, condition (3) is not satisfied for some $y \in \Omega \backslash V$.

$1^{\circ}$ If $\varepsilon \in[0,4 / 13)$, then set $y=1$. It is easy to see that $G(V, y)=\{u \in$ $\mathbb{R}: u>0\}$, so $G(V, y)^{*}=\left\{x^{\prime} \in \mathbb{R}: x^{\prime} \geq 0\right\}$. On the other hand,

$$
\varphi_{\varepsilon} \circ g\left(t x_{0}+(1-t) y\right)-\varphi_{\varepsilon} \circ g(y)<0 \quad \text { for all } t \in(0,1] \text {, }
$$

so $\partial\left(\varphi_{\varepsilon} \circ g\right)(V, y) \subset\left\{x^{\prime} \in \mathbb{R}: x^{\prime}<0\right\}$, hence $\partial\left(\varphi_{\varepsilon} \circ g\right)(V, y) \cap G(V, y)^{*}=\emptyset$. 
$2^{\circ}$ If $\varepsilon \in[4 / 13,1]$, then set $y=2$. Since $G(V, y)=\{u \in \mathbb{R}: u<0\}$ we have $G(V, y)^{*}=\left\{x^{\prime} \in \mathbb{R}: x^{\prime} \leq 0\right\}$. On the other hand,

$$
\varphi_{\varepsilon} \circ g\left(t x_{0}+(1-t) y\right)-\varphi \circ g(y)<0 \quad \text { for all } t \in(0,1],
$$

so $\partial\left(\varphi_{\varepsilon} \circ g\right)(V, y) \subset\left\{x^{\prime} \in \mathbb{R}: x^{\prime}>0\right\}$, hence $\partial\left(\varphi_{\varepsilon} \circ g\right)(V, y) \cap G(V, y)^{*}=\emptyset$.

REMARK 3.5. When $Z$ is one-dimensional a stronger result than Theorem 3.1 is available. For simplicity we assume a continuity condition slightly stronger than necessary.

Proposition 3.6. Let $X$ be a locally convex topological vector space and $\Omega$ be a nonempty subset of $X$. Assume that $g: \Omega \rightarrow \mathbb{R}$ is upper semicontinuous on $\Omega$. Assume that $V$ is a sequentially compact convex subset of $\Omega$ such that $\overline{\Omega \backslash V} \subseteq \Omega$. Then $g$ attains its maximum over $\Omega$ at some point of $V$ if and only if

$$
\emptyset \neq \partial g(V, y) \cap G(V, y)^{*} \quad \text { for every } y \in \Omega \backslash V .
$$

P r o of. First, suppose that $g$ attains a global maximum over $\Omega$ at some $v \in V$. Then

$$
g(v)-g(y) \geq 0 \quad \text { for all } y \in \Omega .
$$

Thus $x_{0}^{\prime}=0 \in X^{\prime}$ satisfies

$$
g(v)-g(y) \geq\left\langle x_{0}^{\prime}, v-y\right\rangle .
$$

By (1), we have $x_{0}^{\prime} \in \partial g(V, y) \cap G(V, y)^{*}$. The sufficiency follows from Theorem 3.1 and Remark 3.2.

In order to present a necessary condition for a vector-valued function $g$ to attain its weak Pareto maximum in a given convex subset $V$ of $\Omega$, we need some definitions.

Recall that

$$
K^{*}=\left\{z^{\prime} \in Z^{\prime}:\left\langle z^{\prime}, k\right\rangle \geq 0 \text { for all } k \in K\right\}
$$

is the dual cone of $K$ and $Z^{\prime}$ is the dual space of $Z$. Define

$$
K_{0}^{*}=\left\{k^{\prime} \in K^{*}:\left\langle k^{\prime}, k_{0}\right\rangle>0 \text { for all } k_{0} \in K^{0}\right\} .
$$

It is easy to show that $K_{0}^{*}=K^{*} \backslash\{0\}$. Clearly, if $k^{\prime} \in K_{0}^{*}$, then $\left\langle k^{\prime}, \cdot\right\rangle$ is strictly monotone with respect to $K^{0}$.

Proposition 3.7. Assume that $X$ and $Z$ are locally convex topological vector spaces and $\Omega$ is a convex subset of $X$. Let $g: \Omega \rightarrow Z$ be a $K$-concave function, and $V$ be a convex subset of $X$. If $g$ attains its weak P-maximum over $\Omega$ in $V$, then there exists $k^{\prime} \in K_{0}^{*}$ such that

$$
\partial\left(k^{\prime} g\right)(V, y) \cap G(V, y)^{*} \neq \emptyset \quad \text { for every } y \in \Omega \backslash V .
$$


Proof. Suppose that $g$ has a weak $P$-maximum at some $v \in V$. Then there is no $y \in \Omega$ such that

$$
-(g(v)-g(y)) \in K^{0} .
$$

By Craven's alternative theorem (see [1, p. 31]), there exists $k^{\prime} \in K_{0}^{*}$ such that

$$
\left\langle k^{\prime}, g(v)-g(y)\right\rangle \geq 0 \quad \text { for all } y \in \Omega .
$$

Thus, $v \in V$ maximizes the linear scalar function $k^{\prime} g$ over $\Omega$. An application of Proposition 3.6 completes the proof.

The following examples show that the main assumptions in Theorem 3.1 and Proposition 3.6 cannot be dropped.

Example 3.8. Define $f: \mathbb{R} \rightarrow \mathbb{R}$ by

$$
f(x)= \begin{cases}0 & \text { for } x \in[0,1) \cup(4,5], \\ x & \text { for } x \in[1,2], \\ 2 & \text { for } x \in(2,3], \\ -x+5 & \text { for } x \in[3,4] .\end{cases}
$$

Let $V=[0,1] \cup[4,5]$. Then $V$ is a sequentially compact non-convex set and $f$ is upper semicontinuous on $\Omega=[0,5]$. Moreover, for all $y \in \Omega \backslash V$ we have $\partial f(V, y) \cap G(V, y)^{*} \neq \emptyset$, since

$$
\begin{aligned}
& \partial f(V, y)=\mathbb{R} \quad \text { and } \quad G(V, y)^{*}=\{0\} \quad \forall y \in(1,2), \\
& \partial f(V, y)=\mathbb{R} \quad \text { and } \quad G(V, y)^{*}=\{0\} \quad \forall y \in(2,3) \text {, } \\
& \partial f(V, y)=\mathbb{R} \quad \text { and } \quad G(V, y)^{*}=\{0\} \quad \forall y \in(3,4) \text {, } \\
& \partial f(V, y)=\mathbb{R} \backslash(0,1) \quad \text { and } \quad G(V, y)^{*}=\{0\} \quad \text { for } y=2 \text {, } \\
& \partial f(V, y)=\mathbb{R} \backslash(-1,0) \quad \text { and } \quad G(V, y)^{*}=\{0\} \quad \text { for } y=3 .
\end{aligned}
$$

However, $f$ does not have a maximum over $\Omega$ in $V$.

Example 3.9. Define $f: \mathbb{R} \rightarrow \mathbb{R}$ by

$$
f(x)= \begin{cases}x & \text { for } x \in(-\infty, 3) \\ 2 & \text { for } x \in[3,+\infty)\end{cases}
$$

(i) Let $\Omega=(-\infty, \infty)$ and $V=[3,4]$. Then $V$ is convex and compact, but $f$ is not upper semicontinuous on $\overline{\Omega \backslash V}$. It is easy to see that $\partial f(V, y) \cap$ $G(V, y)^{*} \neq \emptyset$ for all $y \in \Omega \backslash V$, since

$$
\begin{aligned}
& \partial f(V, y)=(-\infty, 1] \quad \text { and } \quad G(V, y)^{*}=\mathbb{R}_{+} \cup\{0\} \quad \forall y \in(-\infty, 3) \text {, } \\
& \partial f(V, y)=[0, \infty) \quad \text { and } \quad G(V, y)^{*}=\mathbb{R}_{-} \cup\{0\} \quad \forall y \in(4, \infty) .
\end{aligned}
$$

However, $f$ does not have a maximum over $\Omega$ in $V$.

(ii) Let $\Omega=(-\infty, 3) \cup\left[3 \frac{1}{2}, 4\right]$ and $V=\left[3 \frac{1}{2}, 4\right]$. Then $V$ is convex and sequentially compact and $f$ is upper semicontinuous on $\Omega$, but $\overline{\Omega \backslash V} \nsubseteq \Omega$. It is easy to see that $\partial f(V, y) \cap G(V, y)^{*} \neq \emptyset$ for every $y \in \Omega \backslash V$, since 
$\partial f(V, y)=(-\infty, 1]$ and $G(V, y)^{*}=[0, \infty)$ for all $y \in(-\infty, 3)$. However, $f$ does not have a maximum over $\Omega$ in $V$.

(iii) Define $f: \mathbb{R} \rightarrow \mathbb{R}$ by

$$
f(x)= \begin{cases}x & \text { for } x \in(-\infty, 3], \\ 2 & \text { for } x \in(3, \infty) .\end{cases}
$$

Let $\Omega=(-\infty, \infty)$ and $V=[5,6]$. Then $V$ is convex and sequentially compact, $f$ is upper semicontinuous on $\Omega, \partial f(V, 3)=(-\infty,-1 / 3)$ and $G(V, 3)^{*}=\mathbb{R}_{+} \cup\{0\}$, i.e. $\partial f(V, 3) \cap G(V, 3)^{*}=\emptyset$. Observe that $f$ does not have a maximum over $\Omega$ in $V$.

EXAmple 3.10. Let $\Omega=\left\{x=\left(t_{i}\right): \sum_{i=1}^{\infty}\left|t_{i}\right|<\infty\right\}$ and $\|x\|=\sum_{i=1}^{\infty}\left|t_{i}\right|$, for $x=\left(t_{i}\right) \in \Omega$. Define $V=\left\{x=\left(t_{i}\right) \in \Omega:\|x\| \leq 1\right.$ and $t_{i} \geq 0$ for all $i=$ $1,2, \ldots\}$. It is easy to see that $V$ is convex but is not sequentially compact. Let $x_{0}=(-2,-1,-1 / 2,-1 / 4, \ldots)$. Consider the sequence $\left\{x_{k}\right\} \subseteq \Omega, x_{k}=$ $\left(t_{i}^{k}\right)$, such that

$$
\begin{aligned}
x_{1} & =\alpha_{1} x_{0}+\left(1-\alpha_{1}\right) e_{1}, \\
x_{2} & =\alpha_{2} x_{1}+\left(1-\alpha_{2}\right) e_{2}, \\
& \vdots \\
x_{n} & =\alpha_{n} x_{n-1}+\left(1-\alpha_{n}\right) e_{n},
\end{aligned}
$$

where $\alpha_{i}=2^{-i}$ for $1,2, \ldots$ and $e_{1}=(1,0,0, \ldots), e_{2}=(0,1,0, \ldots)$ and so on. Clearly, $e_{i} \in V$ for all $i$, and consequently, $x_{n+1} \in \operatorname{conv}\left(x_{n}, V\right)$. It is easy to see that $\left\{x_{n}\right\} \subset \Omega \backslash V$ because

$$
t_{1}^{n}=-(1 / 2)^{(n-1)(n+2) / 2}<0
$$

and obviously $\left\|x_{n}\right\|<\infty$ for all $n \in \mathbb{N}$. Define $f: \Omega \rightarrow \mathbb{R}$ by

$$
f(x)= \begin{cases}0, & x \neq x_{i} \\ i, & x=x_{i}\end{cases}
$$

Now we show that the sequence $\left\{x_{k}\right\}$ has no cluster points. Indeed, for fixed $i \in \mathbb{N}, t_{i}^{k} \rightarrow 0$ as $k$ tends (no matter how) to infinity. So the only cluster point of $\left\{x_{k}\right\}$ might be $e_{0}=(0,0, \ldots)$. On the other hand,

$$
\left\|x_{k}\right\|=\sum_{i=1}^{\infty}\left|t_{i}^{k}\right| \geq\left|t_{k}^{k}\right| \underset{k \rightarrow \infty}{\longrightarrow} 1 .
$$

This shows that $\left\{x_{k}\right\}$ has no cluster points and, consequently, $f$ is upper semicontinuous on $\Omega$. Finally, we verify condition (3). For $y \notin\left\{x_{k}\right\}$, (3) is satisfied because $e_{0}^{\prime}=(0,0, \ldots) \in G(V, y)^{*} \cap \partial f(V, y)$. The same is true when $y=x_{n}$ for some $n \in \mathbb{N}$. Indeed, $e_{0}^{\prime} \in G\left(V, x_{n}\right)^{*}$ by definition and

$$
f\left(x_{n+1}\right)-f\left(x_{n}\right)=1>\left\langle e_{0}^{\prime}, x_{n+1}-x_{n}\right\rangle,
$$


which together with the decomposition

$$
x_{n+1}=\alpha_{n+1} x_{n}+\left(1-\alpha_{n+1}\right) e_{n+1}
$$

implies that $e_{0}^{\prime} \in \partial f\left(V, x_{n}\right)$. Hence (3) is satisfied for all $y \in \Omega \backslash V$. However, the maximum of $f$ over $\Omega$ is not attained in $V$.

REMARK 3.11. Example 3.10 is related to the drop property. Since $V$ is a closed unit ball, the sequence $\left\{x_{n}\right\}$ has a convergent subsequence if and only if the norm $\|\cdot\|$ has the drop property (see [7], Proposition 2). It is well known that the space $\Omega$ is not reflexive, so $\Omega$ does not have the drop property, and consequently, $\left\{x_{n}\right\}$ may not have a convergent subsequence (cf. [7]).

\section{References}

[1] B. D. Craven, Mathematical Programming and Control Theory, Halsted, London, 1982.

[2] R. Engelking, General Topology, Monograf. Mat. 60, PWN, Warszawa, 1977.

[3] L. Gajek and D. Zagrodny, Existence of maximal points with respect to ordered bipreference relations, J. Optim. Theory Appl. 70 (1991), 355-364.

[4] - - - Countably orderable sets and their applications in optimization, Optimization 26 (1992), 287-301.

[5] _ - - Weierstrass theorem for monotonically semicontinuous functions, ibid. 29 (1994), 199-203.

[6] B. Pshenichny (in Russian).

[7] S. Rolewicz, On drop property, Studia Math. 85 (1987), 27-35.

[8] W. Rudin, Functional Analysis, Moscow, Mir, 1975 (in Russian); English original: McGraw-Hill, New York, 1973.

[9] C. Swartz, Pshenichnyı̆'s theorem for vector minimization, J. Optim. Theory Appl. 53 (1987), 309-317.

Agnieszka Drwalewska and Lesław Gajek

Institute of Mathematics

Technical University of Lódź

Al. Politechniki 11

90-924 Łódź, Poland

E-mail: gal@ck-sg.p.lodz.pl 